\title{
The blood-brain barrier
}

\author{
S Dingezweni
}

Department of Anaesthesia, Charlotte Maxeke Johannesburg Academic Hospital, University of the Witwatersrand, South Africa Corresponding author, email: sdingezweni@ymail.com

\section{Summary}

The blood-brain barrier (BBB) is a dynamic barrier essential for central nervous system interstitial fluid separation from circulating blood. This dynamic separation ensures maintenance of neuronal microenvironment homeostasis against that of the everchanging in solutes and toxin concentration in circulating blood. The blood-brain barrier structure is complex, it has multiple contributors, such as specialised blood microvascular endothelium, neurons, astrocytes and pericytes. Transfer of essential nutrients to the brain and waste products from the brain to circulating blood is tightly regulated and facilitated by a large surface area and specialised transport systems. It is not only the physical characteristics of the barrier that assist in maintenance of neuronal microenvironment, biochemical substances and the high trans endothelial electrical resistance also play a major role.

Circumventricular organs are those parts of the central nervous system lacking the blood-brain barrier. These are essential for optimum central nervous system interaction with circulating blood directly or using neurotransmitters.

Primary or secondary central nervous system pathological states, such as infective and noninfective causes, directly or indirectly induce biochemical mediators that may disrupt and alter blood-brain barrier structure and function.

Understanding of the blood-brain barrier anatomy and physiology assists in developing treatment methods to overcome degenerative and pathological states negatively affecting the central nervous system.

Keywords: blood-brain barrier, tight junctions, microenvironment

\section{Introduction}

To maintain normal brain function, the neural environment must be preserved within a narrow homeostatic range. This requires a tight regulation of transportation of cells, molecules and ions between the blood and the brain.' Such tight regulation is maintained by a unique anatomical and physiological barrier formed collectively in the central nervous system (CNS). The three CNS barrier layers that contribute to the neural tissue, blood and cerebrospinal fluid separation are:

- A highly specialised endothelial cell (EC) layer comprising of the BBB separating the blood and brain interstitial fluid.

- The blood-cerebrospinal fluid barrier (BCSFB) separates blood from the choroid plexus epithelium which secretes the specialised cerebrospinal fluid (CSF) into the cerebral ventricles.

- The arachnoid epithelium separating the blood from the subarachnoid CSF. ${ }^{2,3}$

Blood-brain barrier discovery and existence has progressed over the years. It was first described by Ehrlich in 1885; he described how dye injection into the blood circulation stained peripheral organs but not the spinal cord and the brain. ${ }^{4}$

Later in 1913, Edwin Goldmann showed that direct injection of trypan blue into the CSF stained cells within the CNS and not in the periphery. Reese and Karnovsky presented a solute exchange barrier between the blood and the brain by means of endothelial tight junction complexes. ${ }^{5}$

Development of the BBB is complex. It lies in the early communications of the embryonic endothelium with neural cells. BBB matures during fetal life and is well formed by birth.,

The BBB is structurally defined as specialised endothelial cells lining the intraluminal side of brain capillaries. ${ }^{6}$

It is a highly regulated interface that separates peripheral circulation and the central nervous system. It is estimated that every neuron has a capillary, human brain total length equals $650 \mathrm{~km}$ and capillary surface area available for molecular transport is as high as $20 \mathrm{~m}^{2} .8,9$

\section{Circumventricular organs}

Parts of the CNS structures lack BBB covering; these are the secretory group (pineal gland, median eminence, neurohypophysis), the sensory organs (lamina terminalis, subfornical organs, area postrema) and subcommissural organs. These are collectively known as circumventricular organs (CVO). ${ }^{10,11}$ Lack of $\mathrm{BBB}$ in these organs helps in their neurons sensing changes in hormones and ion concentration changes in CSF and blood stream. 


\section{Structural components of BBB}

Components of BBB are neurons, astrocytes, microglia, pericytes and blood vessels with specialised endothelial cells. The intimate contact between the above components and the functional interactions and signalling between them form a dynamic functional unit, known as the neurovascular unit. ${ }^{12}$

\section{Neurons}

Neurons need a constant supply of oxygen and nutrients within 8-20 $\mu \mathrm{m}$ of distance from capillaries. The brain homeostasis is critical and necessary because neurons are sensitive to many compounds and to subtle changes in their concentrations. Neurons are also part of the neurovascular unit as their terminals arrive to all cells forming the BBB. ${ }^{13}$

\section{Endothelial cells}

Brain microvascular endothelial cells (BMVEC) are situated at the interface between the blood and the brain. They perform essential biological functions including barrier, transport of micro/macronutrients, receptor mediated signalling, leucocyte trafficking and osmoregulation. ${ }^{14}$ The BMVEC structural components include:

- Tight junctions (TJ) composed of TJ proteins (occludin, claudins, zonula occludens (ZO-1, ZO-2, ZO-3, cingulin, AF6, 7H6).

- Adherent junctions (AJ) are composed of cadherin, catenins, vinculin and actin.

- Junctional adhesion molecules that belong to the immunoglobulin superfamily. 13,14

The high trans-endothelial electrical resistance (TEER) in brain endothelium of more than $2000 \mathrm{Ohm} . \mathrm{cm}^{2}$ compared with that of 2-20 Ohm. $\mathrm{cm}^{2}$ resistance in peripheral capillaries is due to a better occlusion of the intercellular cleft by the TJ complex than in peripheral capillaries. TJ integrity depends highly on intact AJ, where the cell-cell adhesion molecules are found. ${ }^{13}$

\section{Astrocytes}

These are glia cells that envelop 99\% of BBB endothelium. Astrocytes are essential for proper neuronal function and astrocyte-BMVE interaction in the neurovascular unit. They are seen as the nerve glue or cement part of the structure. Astrocytes also play a role in sonic hedgehog signalling in the brain which suppresses expression of inflammatory mediators in the brain endothelial cells. ${ }^{15}$

\section{Pericytes}

These are flat, undifferentiated, contractile connective tissue cells that develop around capillary walls. Association of pericytes to blood vessels has been suggested to regulate endothelial cell proliferation, survival, migration, differentiation, and vascular branching.

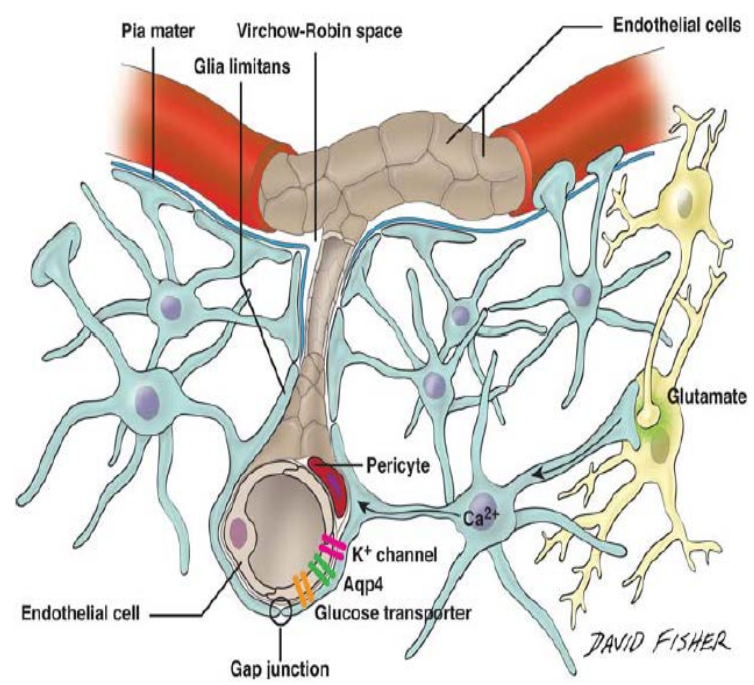

Figure 1: Schematic drawing of the blood-brain barrier and its cross sectional view ${ }^{11}$

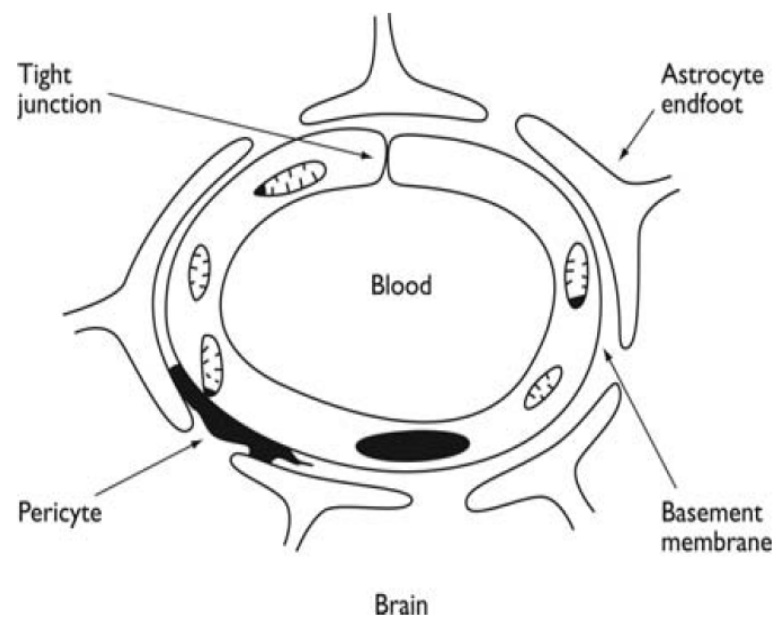

Figure 2: Blood-brain barrier cross sectional view ${ }^{12}$

\section{Transport mechanisms across BBB}

Essential substances cross the BBB via paracellular or transcellular pathways. This transport is strictly limited through both physical (tight junctions) and metabolic barriers (enzymes, diverse transport systems). Nutrients, ions and other molecules cross the BBB by paracellular diffusion through the junctional complex or by the transcellular pathway across the cells. Other factors that determine how easily the substance will passively diffuse through BBB include size less than $180 \mathrm{Da}$, fewer than 10 hydrogen bonds and lipid-soluble molecules. ${ }^{16}$

There are specific transporters that bring in essential nutrients. Essential nutrients, like glucose, are carried across the BBB by slc2a1 also known as GLUT1. Other transporters include slc16a1 for lactate and pyruvate, slc7a1 for cationic amino acids, and slc7a5 for neutral amino acids and L-DOPA.,13

\section{Blood-brain barrier and pathological states}

Infectious (HIV, meningitis) and non-infectious (Parkinson's disease, trauma, stroke, epilepsy, multiple sclerosis, Alzheimer's, tumours - primary and metastatic) pathological states induce 


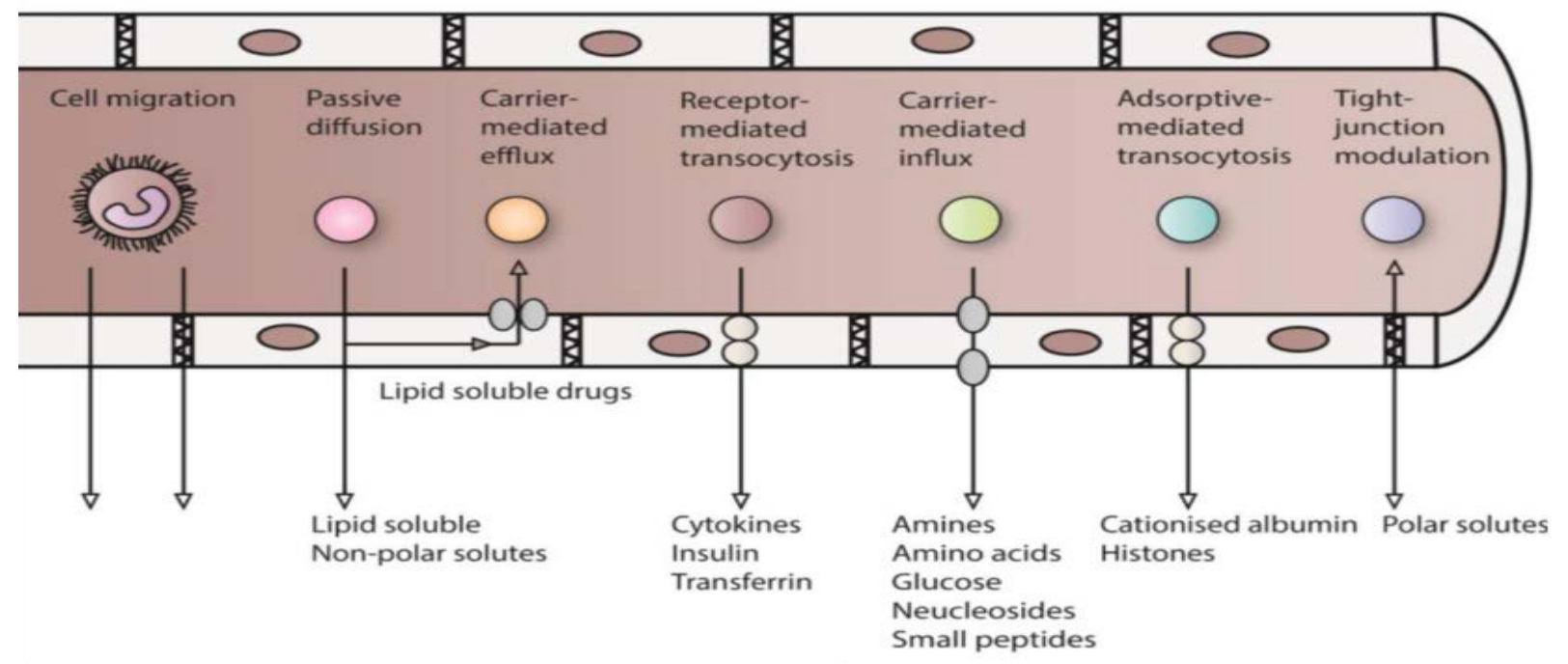

Figure 3: Schematic presentation of different transport mechanisms across the blood-brain barrier ${ }^{4}$

changes in biochemical substance concentration that disrupt BBB integrity. ${ }^{9}$ The following agents are toxic substances that may interrupt BBB integrity and impair its function:

- Bradykinin, histamine, serotonin, glutamate purine nucleotides: ATP, ADP, AMP, adenosine, platelet-activating factor, phospholipase A2, arachidonic acid, prostaglandins, leukotrienes.

- Interleukins: IL-1 a, IL-1 $\beta$, IL-6, tumour necrosis factor-a (TNF- $\alpha$ ), macrophage-inhibitory proteins (MIP1 and MIP2).

- Complement-derived polypeptide C3a-desArg, free radicals, nitric oxide. ${ }^{9}$

\section{Conclusion}

BBB is not the only CNS barrier known. BBB has barrier and carrier functions but not all brain surfaces are covered by this barrier. Enhanced BBB permeability is an invariable response to a primary brain insult and is commonly associated with a variety of brain injuries. Specific pathological changes at the BBB such as opening of the endothelial tight junctions, enhanced transcytosis, damage to the extracellular matrix, upregulated transmembrane water transport, changes in nutrient transport, and pore formation lead to barrier failure.

Understanding BBB physiology and pathological state assists in developing drug delivery methods in order to overcome the difficulties in CNS penetration.

\section{Conflict of interest}

The author declares no conflict of interest.

\section{Funding source}

None.

\section{ORCID}

S Dingezweni (iD https://orcid.org/0000-0002-9300-7724

\section{References}

1. Nicholson C. Diffusion and related transport mechanisms in brain tissue. Rep Prog Phys. 2001;64(7):815. https://doi.org/10.1088/0034-4885/64/7/202.

2. Abbott NJ. Evidence for bulk flow of brain interstitial fluid: significance for physiology and pathology. Neurochem Int. 2004;45(4):545-52. https://doi. org/10.1016/j.neuint.2003.11.006.

3. Abbott NJ, Patabendige AA, Dolman DE, Yusof SR, Begley DJ. Structure and function of the blood-brain barrier. Neurobiol Dis. 2010;37(1):13-25. https://doi. org/10.1016/j.nbd.2009.07.030.

4. Serlin Y, Shelef I, Knyazer B, Friedman A. Anatomy and physiology of the blood-brain barrier. Semin Cell Dev Biol. 2015;38:2-6. https://doi.org/10.1016/j. semcdb.2015.01.002.

5. Reese TS, Karnovsky MJ. Fine structural localization of a blood-brain barrier to exogenous peroxidase. J Cell Biol. 1967;34(1):207-17. https://doi.org/10.1083/ jcb.34.1.207.

6. Stewart PA, Wiley MJ. Developing nervous tissue induces formation of blood-brain barrier characteristics in invading endothelial cells: a study using quail-chick transplantation chimeras. Dev Biol. 1981;84(1):183-92. https://doi. org/10.1016/0012-1606(81)90382-1.

7. Saunders NR, Liddelow SA, Dziegielewska KM. Barrier mechanisms in the developing brain. Front Pharmacol. 2012;3:46. https://doi.org/10.3389/ fphar.2012.00046.

8. Brown PD, Davies SL, Speake T, Millar ID. Molecular mechanisms of cerebrospinal fluid production. Neuroscience. 2004;129(4):955-68. https://doi.org/10.1016/j. neuroscience.2004.07.03.

9. Abbott NJ, Ronnback L, Hansson E. Astrocyte-endothelial interactions at the blood-brain barrier. Nat Rev Neurosci. 2006;7(1):41. https://doi.org/10.1038/ nrn1824.

10. Ufnal $M$, Skrzypecki J. Blood borne hormones in a cross-talk between peripheral and brain mechanisms regulating blood pressure, the role of circumventricular organs. Neuropeptides. 2014;48(2):65-73. https://doi.org/10.1016/j. npep.2014.01.003.

11. Sharif $Y$, Jumah $F$, Coplan L, et al. Blood brain barrier: a review of its anatomy and physiology in health and disease. Clin Anat. 2018;31(6):812-23.

12. Lawther BK, Kumar S, Krovvidi H. Blood-brain barrier. BJA Educ. 2011;11(4):12832. https://doi.org/10.1093/bjaceaccp/mkr018.

13. Tajes M, Ramos-Fernandez E, Weng-Jiang $X$, et al. The blood-brain barrier: structure, function and therapeutic approaches to cross it. Mol Membr Biol. 2014;31(5):152-67. https://doi.org/10.3109/09687688.2014.937468.

14. Persidsky Y, Ramirez SH, Haorah J, Kanmogne GD. Blood-brain barrier: structural components and function under physiologic and pathologic conditions.J Neuroimmune Pharmacol. 2006;1(3):223-36. https://doi.org/10.1007/ s11481-006-9025-3.

15. Sweeney MD, Zhao Z, Montagne A, Nelson AR, Zlokovic BV. Blood-brain barrier: from physiology to disease and back. Physiol Rev. 2019;99(1):21-78. https://doi. org/10.1152/physrev.00050.2017.

16. Pardridge WM. Alzheimer's disease drug development and the problem of the blood-brain barrier. Alzheimers Dement. 2009;5(5):427-32. https://doi. org/10.1016/j.jalz.2009.06.003. 